\title{
Músculo Plantar en Individuos Brasileños
}

\author{
Plantaris Muscle in Brazilian Individuals
}

"Luiz Carlos Buarque de Gusmão; ** Célio Fernando de Sousa Rodrigues \& ${ }^{* * * *}$ Josefa Sileda Martins

GUSMÃO, L. C. B.; SOUSA-RODRIGUES, C. F. \& MARTINS, J. S. Músculo plantar en individuos brasileños.Int. J. Morphol., 29(2):344-346, 2011.

RESUMEN: El objetivo del trabajo fue obtener información más detallada sobre el músculo plantar, que permita mayor comprensión sobre su presencia, morfología, inserción, trayecto de su tendón y la extensión del vientre muscular y del tendón, facilitando su retirada para utilizarlo en injertos. Fueron realizadas disecciones de 60 miembros inferiores de cadáveres adultos fijados en formaldehído al 10\%. El músculo plantar estuvo presente en 57/60 casos (95\%). La forma de huso del músculo fue encontrada en $100 \%$ de los casos. La longitud del vientre muscular varió entre 7,0 cm y 13,7 cm. La extensión del tendón varió entre 25,5 y 40,0 cm. El trayecto del tendón fue oblicuo en $82,46 \%$, entre los músculos sóleo y gastrocnemio, ubicándose en el resto del trayecto medialmente al tendón calcáneo. En 17,54\% de los casos el tendón del músculo plantar, tras cruzar entre los músculos gastrocnemio y sóleo, se separó de éstos para seguir anterior al tendón calcáneo. El tendón se insertó en el 79 \% en la cara medial del tendón calcáneo. En el 21\% de los casos, la inserción se realizó en diversos sitios como: cara anterior del tendón calcáneo; retináculo de los extensores o de los músculos flexores; en el calcáneo; en el músculo sóleo y en la cápsula de la articulación talocrural.

\section{PALABRAS CLAVE: Anatomía; Miembro inferior; Músculo plantar; Ortopedia; Cirugía Plástica.}

\section{INTRODUCCIÓN}

En la reparación quirúrgica de las lesiones tendinosas se utilizan injertos obtenidos de tendones de músculos, cuya retirada no causa perjuicio funcional en la zona del área donada. El músculo plantar es uno de los que más se presta a este fin, pues además de tener una función insignificante, su largo tendón permite el injerto en tendones de músculos más nobles (Moore, 1994).

Las descripciones de la literatura anatómica sobre el músculo plantar son escasas, limitándose en la mayor parte de los casos apenas citarlo. La presente investigación tiene por objeto obtener informaciones más detalladas sobre este músculo que puedan otorgar una mayor comprensión sobre su morfología. El conocimiento de la extensión de su vientre muscular y de su tendón, el trayecto del tendón y su inserción, dan un mejor conocimiento anatómico sobre su real contribución para la flexión plantar, permitiendo que el abordaje quirúrgico para la retirada del tendón sea realizado con más seguridad y sin daños a las estructuras vecinas.

\section{MATERIAL Y MÉTODO}

Fueron disecados 60 miembros inferiores, (30 izquierdos y 30 derechos) de cadáveres adultos, de ambos sexos, fijados en formaldehído al $10 \%$ y pertenecientes al laboratorio de Anatomía Humana de la Universidade Federal de Alagoas y del Centro Anatómico del Instituto Batista de Enseñanza Superior de Alagoas, Brasil. En virtud que los cadáveres fueron donados por el Instituto Médico Legal Estácio de Lima para la Universidad Federal de Alagoas, cumpliendo lo que determina la Ley Federal 8.501, no se realizó el Consentimiento informado.

\section{RESULTADOS}

El músculo plantar se presentó siempre fusiforme y estaba ausente en 3 casos ( $5 \%$ ), en dos casos esta ausencia fue bilateral. La longitud del vientre muscular varió entre $7,0 \mathrm{~cm}$ a $13,7 \mathrm{~cm}$ en el lado derecho y de $7,0 \mathrm{~cm}$ a $13,5 \mathrm{~cm}$

\footnotetext{
Profesor de Anatomía Humana de La Universidad Federal de Alagoas y del Instituto Batista de Enseñanza Superior de Alagoas. Máster y Doctor en Anatomía Humana. Miembro Titular del Colegio Brasileño de Cirujanos, Brasil.

** Profesor de Anatomía Humana de la Universidad Federal de Alagoas y de la Universidad e Ciencias y de la Salud de la Provincia de Alagoas. Máster y Doctor en Anatomía Humana, Brasil.

*** Profesora de Anatomía del Instituto Batista Superior de Alagoas. Especialista en Educación Física en Ambientes Escolar y no Escolar. Maestranda en Ciencias de la Salud por la Universidad Federal de Alagoas, Brasil.
} 
en el lado izquierdo constatando un valor promedio de 10,90 $\mathrm{cm}$. El tendón del músculo acompañaba el margen medial del tendón del músculo tríceps sural (tendón calcáneo o tendón de Aquiles) y su extensión tuvo una variación entre 25,5 $\mathrm{cm}$ a $38,8 \mathrm{~cm}$ en el lado derecho y de $26,7 \mathrm{~cm}$ a $40,0 \mathrm{~cm}$ en el lado izquierdo, con un valor promedio de $31,88 \mathrm{~cm}$. En 50/57 (87,72\%) la inserción del músculo plantar se realizó en la cara medial o anterior del tendón calcáneo.En los otros siete casos, la inserción se realizó: directamente en el calcáneo (tres casos); en el retináculo de los músculos extensores (un caso); en el retináculo de los músculos flexores (un caso); en la cápsula de la articulación talocrural (un caso) y en el tendón del músculo sóleo, antes de que éste se funda con los gastrocnemios (un caso).

\section{DISCUSIÓN}

La ausencia del músculo plantar en aproximadamente
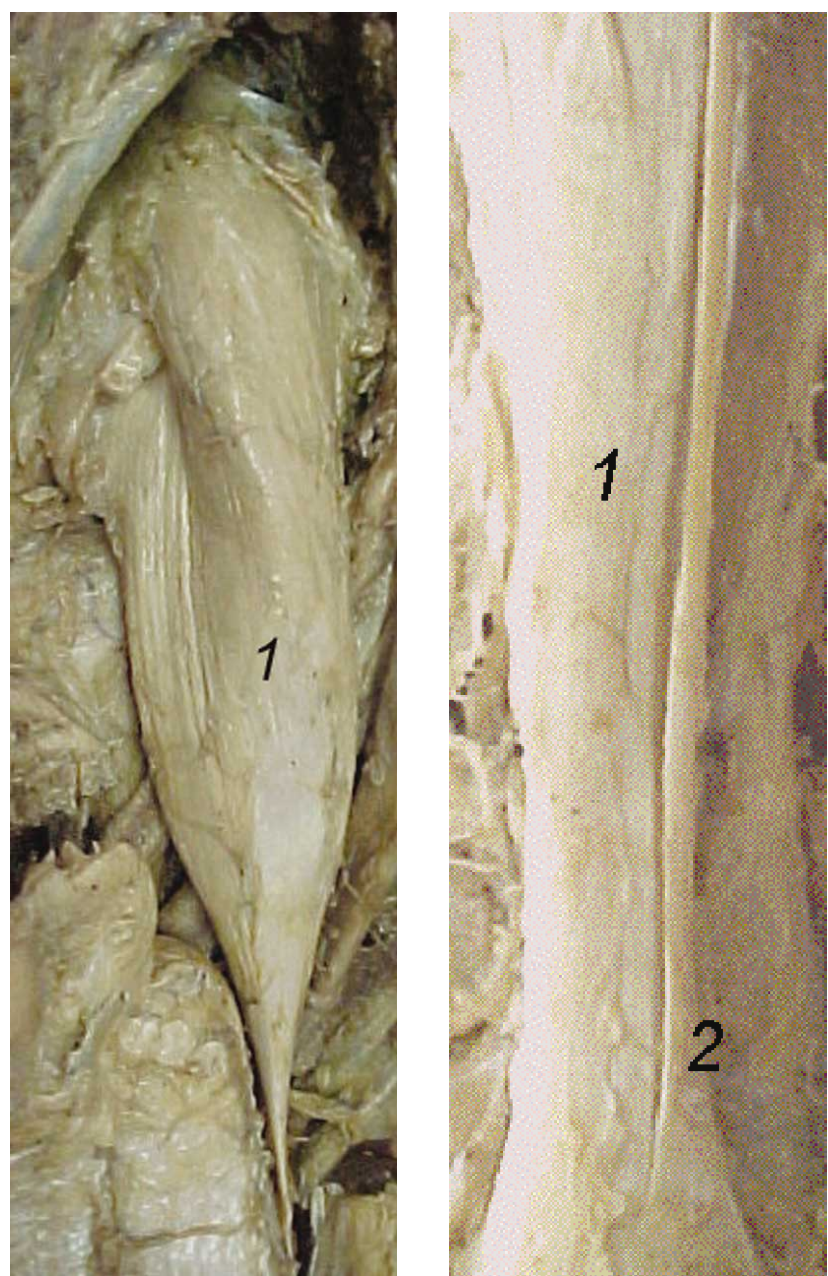

Fig. 1. Cara posterior de la pierna. 1 Músculo plantar. 2. Tendón del músculo plantar. el 5\% ha sido la más baja encontrada en comparación con los hallazgos de Gruber in Le Double (1897), quien reportó la ausencia en el 7,5\%, y los de Schwalbe \& Pfitzner in Le Double, de 6,3\% en hombres y 5,9\% en mujeres. Por otra parte, Rasch (1991) señaló la ausencia del músculo plantar en $8 \%$.

No han sido encontrados músculos con longitud de 5,0 cm como han informado Testut \& Latarjet (1959), Hollinshead (1966) y Goss (1988). En nuestras observaciones el menor valor fue de $7,0 \mathrm{~cm}$. Con respecto al valor máximo de 10,0 cm citado por Hollinshead, Woodburne (1978) y Goss de $12,0 \mathrm{~cm}$ citado por Testut \& Latarjet, están dentro de los descritos en nuestro estudio.

Con respecto a la longitud del tendón del músculo plantar no ha sido citado en la literatura. Creemos que se trata de un dato importante, no valorado por los autores, pues en los procedimientos quirúrgicos, con retirada del tendón para injerto en tendones de otros músculos, la información sobre la longitud resulta de vital importancia, permitiendo verificar si existe suficiente tendón para la reparación de la lesión en el área receptora. En nuestras observaciones, el tendón del músculo plantar tuvo una extensión que variaba entre $25,5 \mathrm{~cm}$ a $40,0 \mathrm{~cm}$, con un valor promedio de $31,88 \mathrm{~cm}$, lo que permite el injerto en la mayor parte de los tendones o en la práctica de injertos múltiples.

La zona donde el tendón puede ser ubicado es muy importante para el cirujano en el momento de su retirada. La precisión de su localización permite que el procedimiento quirúrgico sea rápido, utilizando pequeñas incisiones y con el mínimo de traumas y de complicaciones. Las relaciones del trayecto del músculo plantar con el tendón del músculo tríceps sural, citadas por Oliveira Neto (1951), Schaffer (1953), Testut \& Latarjet, Gardner et al. (1978), Woodburne, Goss, Latarjet \& Ruiz Liard (1993), Moore y Palastanga et al. (2000), han señalado que el tendón del músculo plantar acompaña el margen medial del tendón del calcáneo, hecho observado en prácticamente todas nuestros casos. El cambio de trayectoria del tendón han ocurrido en el tercio inferior de la pierna y se debe mucho más a variaciones anatómicas locales que a alteraciones de trayecto.

Con respecto a la inserción del tendón, hay una heterogeneidad de afirmaciones, hecho concordante con nuestros resultados. Sin embargo, la inserción en las caras medial o anterior del tendón calcáneo, alrededor del 87,72\% han sido las observaciones más comúnmente encontradas por Testut \& Latarjet, Hollinshead, Gardner et al., Latarjet \& Ruiz Liard, Goss y Rasch. Con respecto a las inserciones atípicas, como en el ligamento colateral medial, citado por Brown in Testut (1884); entre el tendón calcáneo y el maléolo 
medial, referido por Sandifort in Testut (1884); en la fascia del tobillo informado por Gardner et al. y anterior y lateral al tendón calcáneo descrito por Woodburne, también han sido encontrados en nuestro estudio. Estos puntos de inserción fuera del tendón calcáneo, correspondieron a 7/57 casos $(12,28 \%)$, como ya fue descrito en el retináculo de los músculos flexores o extensores; en la cápsula de la articulación talocrural; en el tendón del músculo sóleo o aislada- mente en el calcáneo. Debemos recordar que a pesar del número significativo de casos, no interfieren con el objetivo principal del trabajo que fue informar la ubicación del tendón, pues la posición de éste en relación al tendón del músculo tríceps sural no se modifica y es independiente del punto de inserción. De esta manera se puede afirmar con seguridad que el tendón estará en 100\% de los casos localizados en el margen medial del tendón calcáneo.

GUSMÃO, L. C. B.; SOUSA-RODRIGUES, C. F. \& MARTINS, J. S. The plantaris muscle in Brazilian individuals.Int. J. Morphol., 29(2):344-346, 2011.

SUMMARY: The aim of this study was to obtain more detailed information on the plantar muscle, which may provide a greater understanding of their presence, their morphology, their distal insertion, the path of its tendon, and the extent of the muscle and tendon, facilitating its removal for use in grafts. We performed 60 dissections of the lower limbs of adult cadavers in formaldehyde $10 \%$. The plantar muscle was present in 57/60 of the cases (95\%). In the $100 \%$ of the cases the muscle has a fusiform shape. The length of the muscle ranged from $7.0 \mathrm{~cm}$ to $13.7 \mathrm{~cm}$. The length of the tendon ranged between $25.5 \mathrm{~cm}$ to $40 \mathrm{~cm}$. The trajectory of the tendon was oblique in $82.46 \%$ between the medial gastrocnemius and soleus muscles, located in the remainder of its trajectory, medially to the calcaneous tendon. In $17.54 \%$ of the remaining cases the plantar tendon, after passing between the gastrocnemius and soleus muscles, it separated to move in the direction of the anterior calcaneal tendon. The muscle had its distal insertion in $79 \%$ on the medial calcaneal tendon. In $21 \%$ remaining cases, the insertion was in various locations such as on the soleus muscle, the flexor retinaculum and joint capsule of ankle joint.

KEY WORDS: Anatomy; Lower limb; Plantar muscle; Orthopedics; Plastic surgery.

\section{REFERENCIAS BIBLIOGRÁFICAS}

Gardner, E.; Gray, D. J. \& O’Rahilly, R. Anatomia Estudo Regional do Corpo Humano. $4^{\mathrm{a}}$ ed. Rio de Janeiro, Guanabara Koogan, 1978. p.227.

Goss, M. C. Anatomia. 29 ed. Rio de Janeiro, Guanabara, 1988. p.416.

Gruber, W. In : Le Doublé, A. F. Traité des Variatones du Susteme Musculaire de L'Homme. Paris, Schleicher Freres, 1897. p.315.

Hollinshead, W. H. Anatomia Humana. Rosário, La Medica, 1966. p.529.

Latarjet, M. \& Ruiz Liard, A. Anatomia Humana. $2^{\mathrm{a}}$ ed., São Paulo, Panamericana, 1993. p.859.

Moore, K. L. Anatomia Orientada para a Clínica. $3^{\mathrm{a}}$ ed. Rio de Janeiro, Guanabara Koogan, 1994. p.407.

Oliveira Neto, P. B. Manual de Dissecação. Rio de Janeiro, Editora Cientifica, 1951. p.140.

Palastanga, N.; Field, D. \& Soams, R. Anatomia e Movimento Humano. Estrutura e Função. $3^{\mathrm{a}}$ ed. São Paulo, Manole, 2000. p.308.
Rasch, P. J. Cinesiologia e Anatomia Aplicada. $7^{\text {a }}$ ed. Rio de Janeiro, Guanabara Koogan, 1991. p.164.

Schaeffer, J. P. Morris' Human Anatomy: A Complete Systematic Treatise. $11^{\mathrm{a}}$ ed. New York, McGraw-Hill, 1953. p.575.

Schwalbe \& Pfeitzer. In: Le Double, A. F. Traité de Variatones du Systeme Musculaire. Paris, Schleicher Freres, 1897. p.315.

Testut, L. Les Malies Musculaires Chez L'Homme: Liquées par L'Anatomie Comparée. Paris, Masson, 1884. p. 659, 666.

Testut, L. \& Latarjet, A. Tratado de Anatomia Humana. 9a ed. Barcelona, Salvat Editores, 1959. p.1165.

Woodburne, R. T. Anatomia Humana. $6^{\text {a }}$ ed. Rio de Janeiro, Guanabara Koogan, 1978. p. 447.

Dirección para correspondencia:

Prof. Dr. Luiz Carlos Buarque de Gusmão

Condomínio Aldebaran Alfa

Quadra F, n 08. Tabuleiro dos Martins

CEP: $57080-900$.

Maceió-AL

BRASIL.

Recibido : 18-10-2010

Aceptado: 23-02-2011 